\title{
Properties of Cosmic Aluminum Nuclei: Results from the Alpha Magnetic Spectrometer
}

\section{Zhen Liu, ${ }^{a, *}$ Mercedes Paniccia ${ }^{a}$ and Cheng Zhang ${ }^{b}$ on behalf of the AMS Collaboration}

(a complete list of authors can be found at the end of the proceedings)

${ }^{a}$ DPNC, Université de Genève,

1211 Genève 4, Switzerland

${ }^{b}$ Institute of High Energy Physics,

Beijing, China

E-mail: zhen.liu@cern.ch, mercedes.paniccia@cern.ch, cheng.zhang@cern.ch

We report the properties of aluminum (Al) nuclei in cosmic rays measured in the rigidity range 2.15 GV to 3.0 TV with 0.51 million nuclei collected by the Alpha Magnetic Spectrometer experiment on the International Space Station. We observed that above $6 \mathrm{GV}$ the $\mathrm{Al}$ spectrum is well described by the weighted sum of the silicon spectrum (primary cosmic rays) and the fluorine spectrum (secondary cosmic rays). The fraction of the primary component increases with rigidity and becomes dominant at highest rigidities. Al/Si abundance ratio at the source is determined independent of cosmic ray propagation.

$37^{\text {th }}$ International Cosmic Ray Conference (ICRC 2021)

July 12th - 23rd, 2021

Online - Berlin, Germany

\footnotetext{
${ }^{*}$ Presenter
} 


\section{Introduction}

Aluminum nuclei in cosmic rays, like nitrogen, are thought to be produced both in astrophysical sources and by the collisions of heavier nuclei with the interstellar medium [1]. Previously, the measurement of the cosmic nitrogen flux with the Alpha Magnetic Spectrometer experiment (AMS) has been reported [2]. Remarkably, the nitrogen flux is well described by the sum of a primary component (proportional to the oxygen flux [2]) and a secondary component (proportional to the boron flux [2]). Recently, AMS also reported the properties of primary heavy cosmic-ray $\mathrm{Ne}, \mathrm{Mg}$, and Si fluxes [2,3] and of heavy secondary cosmic-ray F flux [4]. The AMS results revealed that there are two classes of primary cosmic rays: that of the light He-C-O nuclei and that of the heavy $\mathrm{Ne}-\mathrm{Mg}-\mathrm{Si}$ nuclei. They also revealed that there are two classes of secondary cosmic rays: that of the light $\mathrm{Li}-\mathrm{Be}-\mathrm{B}$ nuclei and that of the heavy $\mathrm{F}$ nuclei.

We present the precise measurement of the $\mathrm{Al}$ flux in the rigidity range from $2.15 \mathrm{GV}$ to 3.0 TV based on 0.51 million aluminum nuclei collected by AMS during its first 8.5 years (from May 19, 2011 to October 30, 2019) of operation on the International Space Station (ISS).

\section{AMS-02 detector and analysis}

AMS is a long duration, large acceptance precision magnetic spectrometer on space which is able to measure the sign and value of the charge, the momentum, and the rigidity of charged particles. The layout and description of the AMS detector are presented in Refs. [2]. The key elements of AMS detector used in the aluminum flux measurement are the permanent magnet, nine layers of the silicon tracker, and four planes of time of flight (TOF) scintillation counters.

Further information on the performance of the rigidity and charge measurements, and the Monte-Carlo (MC) simulations, the flux analysis procedure including the event selection, background subtraction, bin-to-bin migration correction, and study of the systematic uncertainties are detailed in Refs. [13].

Several independent analyses were performed on the same data sample by different study groups. As shown in Fig. 1, the results of those analyses are consistent.

\section{Properties of aluminum cosmic ray nuclei}

Fig. 2 shows the AMS aluminum flux as a function of rigidity $\tilde{R}$ with the total errors, together with the AMS nitrogen flux [2]. As seen, at rigidities above $~ 100 \mathrm{GV}$ the $\mathrm{Al}$ flux and the $\mathrm{N}$ flux have similar rigidity dependence.

Fig. 3 shows the AMS aluminum flux as a function of kinetic energy per nucleon $E_{K}$ together with earlier measurements [5-10]. Data from other experiments have been extracted using Ref. [11]. Also shown in the figure are the predictions of the latest GALPROP-HELMOD cosmic ray propagation model [12] based on published AMS data. The GALPROP-HELMOD model agrees well with the AMS aluminum data above $3 \mathrm{GeV} / \mathrm{n}$.

To obtain the primary $\Phi_{\mathrm{Al}}^{\mathrm{P}}$ and secondary $\Phi_{\mathrm{Al}}^{\mathrm{S}}$ components in the $\mathrm{Al}$ spectrum $\Phi_{\mathrm{Al}}=\Phi_{\mathrm{Al}}^{\mathrm{P}}+\Phi_{\mathrm{Al}}^{\mathrm{S}}$, a fit of $\Phi_{\mathrm{Al}}$ to the weighted sum of a heavy primary cosmic ray spectrum, namely silicon $\Phi_{\mathrm{Si}}$ [3], and of a heavy secondary cosmic ray spectrum, namely fluorine $\Phi_{\mathrm{F}}[4]$, was performed above $6 \mathrm{GV}$. 


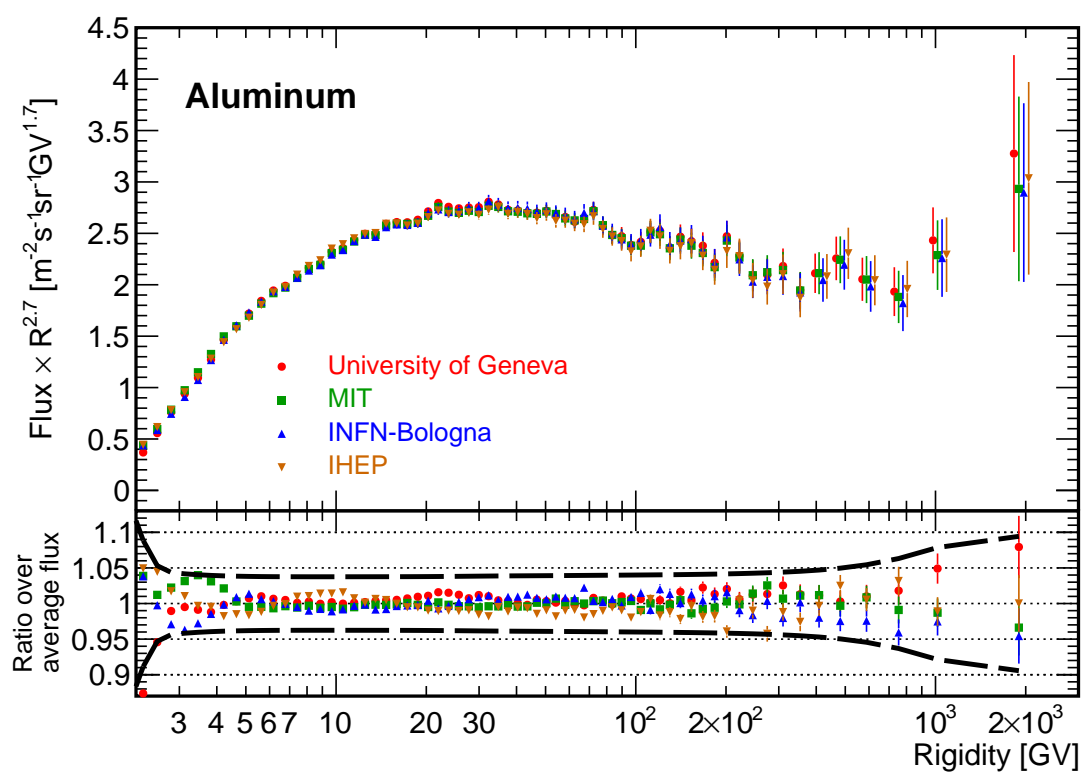

Figure 1: The AMS aluminum (Al) flux multiplied by $\tilde{R}^{2.7}$ as a function of rigidity (upper panel) and the ratio over average flux (lower panel) obtained on the same data sample by four independent study groups from the University of Geneva (red dots), MIT (green squares), INFN-Bologna (blue triangles) and IHEP (brown triangles). The correlation in the flux error from the statistics has been subtracted. The dashed black lines in the lower panel show the average of the systematical error.

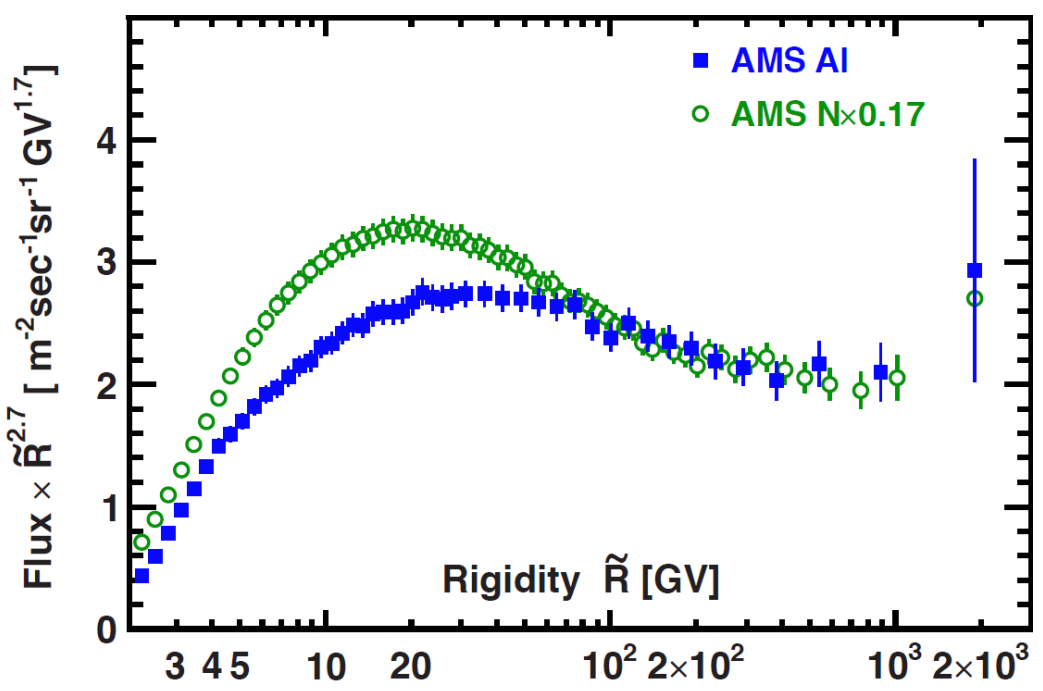

Figure 2: The AMS aluminum (Al) flux together with the rescaled AMS nitrogen (N) flux [2] multiplied by $\tilde{R}^{2.7}$ with total errors as function of rigidity.

The fit yields $\Phi_{\mathrm{Al}}^{\mathrm{P}}=(0.103 \pm 0.004) \times \Phi_{\mathrm{Si}}$ and $\Phi_{\mathrm{Al}}^{\mathrm{S}}=(1.04 \pm 0.03) \times \Phi_{\mathrm{F}}$ with $\chi^{2} /$ d.o.f $=24 / 36$, as shown in Fig. 4.

As seen from Fig. 4, the contributions of the primary component increases with rigidity. The same dependence was also observed for the spectra of nitrogen $\Phi_{N}$ [2] and sodium $\Phi_{\mathrm{Na}}$ [13]. 


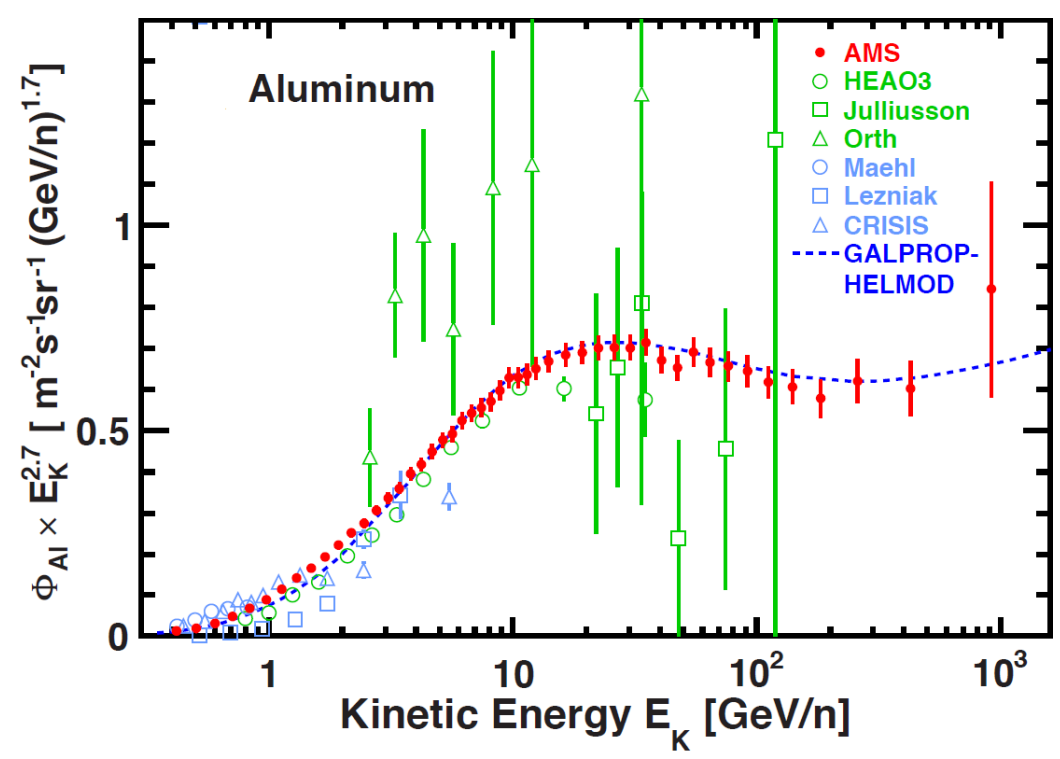

Figure 3: The AMS aluminum (Al) flux as functions of kinetic energy per nucleon $E_{K}$ multiplied by $E_{K}^{2.7}$ together with earlier measurements [5-10]. For the AMS measurement the rigidity has been converted to kinetic energy per nucleon as $E_{K}=\left(\sqrt{Z^{2} \tilde{R}^{2}+M^{2}}-M\right) / A$ where $Z, M$ and $A$ are the ${ }_{13}^{27} \mathrm{Al}$ nuclear charge, mass and atomic mass number, respectively. The dashed blue line show the prediction of the latest GALPROP-HELMOD [12] model based on published AMS data on two primary cosmic ray classes, He-C-O and $\mathrm{Ne}-\mathrm{Mg}-\mathrm{Si}$ and other AMS data. Note the latest GALPROP-HELMOD model agrees well with the AMS aluminum data above $3 \mathrm{GeV} / \mathrm{n}$.

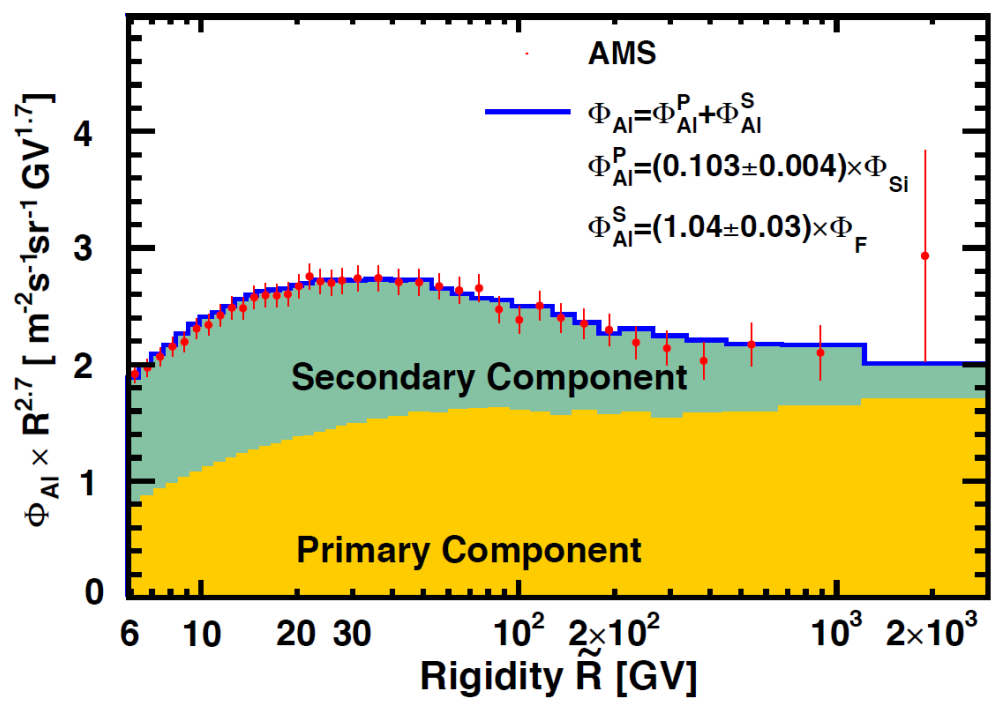

Figure 4: The AMS aluminum flux $\Phi_{\mathrm{Al}}$ fit to the weighted sum of the silicon flux $\Phi_{\mathrm{Si}}$ and the fluorine flux $\Phi_{\mathrm{F}}$ above $6 \mathrm{GV}$, i.e. $\Phi_{\mathrm{Al}}=\Phi_{\mathrm{Al}}^{\mathrm{P}}+\Phi_{\mathrm{Al}}^{\mathrm{S}}$. The contributions of the primary and secondary components are indicated by the shading (yellow and green, respectively).

The observation that, similar to $\mathrm{N}$ and $\mathrm{Na}$, the $\mathrm{Al}$ spectrum can be fit over a wide rigidity range as the linear combination of primary and secondary spectrum permits the direct determination of the $\mathrm{Al} / \mathrm{Si}$ abundance ratio at the source, $0.103 \pm 0.004$, without the need to consider the Galactic 
propagation of cosmic rays.

Figure 5 presents cosmic nuclei fluxes measured by AMS as functions of rigidity from $\mathrm{He}$ $(Z=2)$ to $\mathrm{Si}(Z=14)$. It shows that there are two classes of primary cosmic rays, He-C-O and $\mathrm{Ne}-\mathrm{Mg}-\mathrm{Si}$, and two classes of secondary cosmic rays, Li-Be-B and $\mathrm{F}$ [4], and that $\mathrm{N}, \mathrm{Na}$, and $\mathrm{Al}$ belong to a distinct group of cosmic rays which are the combinations of primary and secondary cosmic rays.

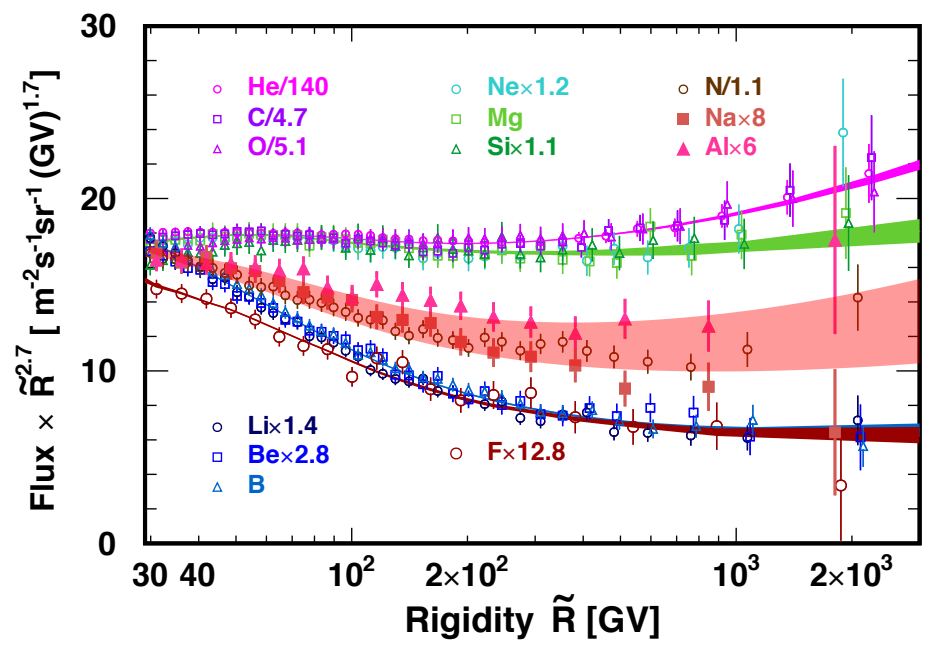

Figure 5: Cosmic nuclei spectra measured by AMS as functions of rigidity from $Z=2$ to $Z=14$ above $30 \mathrm{GV}$. For clarity, data points above $400 \mathrm{GV}$ are displaced horizontally. For display purposes only, fluxes were rescaled as indicated. The shaded tan band on $\mathrm{N}, \mathrm{Na}$, and $\mathrm{Al}$ is to guide the eye.

\section{Conclusion}

We have presented the precision measurement of the $\mathrm{Al}$ flux as a function of rigidity from $2.15 \mathrm{GV}$ to 3.0 TV based on the data collected by AMS during its first 8.5 years operation.

We found that $\mathrm{Al}$ nuclei, together with $\mathrm{N}$ and $\mathrm{Na}$, belong to a distinct group of cosmic rays which are combinations of primary and secondary cosmic rays. Similar to $\mathrm{N}$ and Na spectra, the $\mathrm{Al}$ spectrum is well described by the sum of a primary cosmic ray component and a secondary cosmic ray component. The fraction of the primary component increases with rigidity and becomes dominant at the highest rigidities. The $\mathrm{Al} / \mathrm{Si}$ abundance ratio at the source $(0.103 \pm 0.004)$ is directly determined independent of cosmic ray propagation. These are new and unexpected properties of cosmic rays.

\section{References}

[1] I. A. Grenier, J. H. Black, and A. W. Strong, The Nine Lives of Cosmic Rays in Galaxies, Annu. Rev. Astron. Astrophys. 53, 199 (2015); P. Blasi, The origin of galactic cosmic rays, Astron. Astrophys. Rev. 21, 70 (2013); A. W. Strong, I. V. Moskalenko, and V. S. Ptuskin, CosmicRay Propagation and Interactions in the Galaxy, Annu. Rev. Nucl. Part. Sci. 57, 285 (2007); 
A. Castellina and F. Donato, Diffusion coefficient and acceleration spectrum from direct measurements of charged cosmic ray nuclei, Astropart. Phys. 24, 146 (2005); G. Jóhannesson et al., BAYESIAN ANALYSIS OF COSMIC RAY PROPAGATION: EVIDENCE AGAINST HOMOGENEOUS DIFFUSION, Astrophys. J. 824, 16 (2016).

[2] M. Aguilar et al., The Alpha Magnetic Spectrometer (AMS) on the International Space Station: Part II - Results from the First Seven Years, Phys. Rep. 894, 1 (2021). Note that in this Letter we have used the He-C-O, Li-Be-B, Ne-Mg-Si, F, and $\mathrm{N}$ data covering the same collection time as $\mathrm{Na}$ and $\mathrm{Al}$.

[3] M. Aguilar et al., Properties of Neon, Magnesium, and Silicon Primary Cosmic Rays Results from the Alpha Magnetic Spectrometer, Phys. Rev. Lett. 124, 211102 (2020).

[4] M. Aguilar et al., Properties of Heavy Secondary Fluorine Cosmic Rays: Results from the Alpha Magnetic Spectrometer, Phys. Rev. Lett. 126, 081102 (2021).

[5] E. Juliusson, Charge Composition and Energy Spectra of Cosmic-Ray Nuclei at Energies above 20 GeV Per Nucleon, Astrophys. J. 191, 331 (1974).

[6] R. Maehl, J. F. Ormes, A. J. Fisher, and F. A. Hagen, Energy spectra of cosmic ray nuclei: $4 \leq Z \leq 26$ and $0.3 \leq E \leq 2 \mathrm{GeV} \mathrm{amu}{ }^{-1}$, Astrophys. Space Science, 47, 163 (1977).

[7] C. D. Orth, A. Buffington, G. F. Smoot, and T. S. Mast, Abundances and spectra for cosmic-ray nuclei from lithium to iron for 2 to $150 \mathrm{GeV}$ per nucleon, Astrophys. J. 226, 1147 (1978).

[8] J. Lezniak and W. Webber, The charge composition and energy spectra of cosmic-ray nuclei from $3000 \mathrm{MeV}$ per nucleon to $50 \mathrm{GeV}$ per nucleon, Astrophys. J. 223, 676 (1978).

[9] J. S. Young, P. S. Freier, C. J. Waddington, N. R. Brewster, and R. K. Fickle, The elemental and isotopic composition of cosmic rays - Silicon to nickel, Astrophys. J. 246, 1014 (1981).

[10] J.J. Engelmann et al., Charge composition and energy spectra of cosmic-ray nuclei for elements from Be to Ni - Results from HEAO-3-C2, Astron. Astrophys. 233, 96 (1990).

[11] D. Maurin, F. Melot, and R. Taillet, A database of charged cosmic rays, Astron. Astrophys. 569, A32 (2014).

[12] M. J. Boschini et al., Inference of the Local Interstellar Spectra of Cosmic-Ray Nuclei $Z \leq 28$ with the GalProp-HelMod Framework, Astrophys. J. Suppl. Ser. 250, 27 (2020).

[13] M. Aguilar et al., Properties of Cosmic Sodium and Aluminum Nuclei: Results from the Alpha Magnetic Spectrometer. Phys. Rev. Lett, 2021. In Press. 


\section{Full Authors List: AMS Collaboration}

M. Aguilar ${ }^{28}$, L. AliCavasonza ${ }^{1}$, B. Alpat ${ }^{35}$, G. Ambrosi ${ }^{35}$, L. Arruda ${ }^{26}$, N. Attig ${ }^{23}$, F. Barao ${ }^{26}$, L. Barrin ${ }^{14}$, A. Bartoloni ${ }^{41}$, S. Başeğmez-du Pree ${ }^{17}$, R. Battiston ${ }^{38,39}$, M. Behlmann ${ }^{9}$, B. Beranek ${ }^{1}$, J. Berdugo ${ }^{28}$, B. Bertucci ${ }^{35,36}$, V. Bindi ${ }^{19}$, K. Bollweg ${ }^{20}$, B. Borgia ${ }^{41,42}$, M.J. Boschini ${ }^{30}$, M.Bourquin ${ }^{15}$, E.F. Bueno ${ }^{17}$, J. Burger ${ }^{9}$, W.J. Burger ${ }^{38}$, S. Burmeister ${ }^{24}$, X.D.Cai ${ }^{9}$, M.Capel1 ${ }^{9}$, J. Casaus ${ }^{28}$, G. Castellini ${ }^{13}$, F.Cervelli ${ }^{37}$, Y.H.Chang ${ }^{46}$, G.M.Chen ${ }^{6,7}$, G.R.Chen ${ }^{20}$, H.S. Chen ${ }^{6,7}$, Y.Chen ${ }^{15}$, L. Cheng ${ }^{20}$, H.Y.Chou ${ }^{47}$, S. Chouridou ${ }^{1}$, V.Choutko ${ }^{9}$, C.H.Chung ${ }^{1}$, C. Clark ${ }^{9}, 20$, G. Coignet $^{3}$, C.Consolandi ${ }^{19}$, A.Contin ${ }^{8}$, C. Corti ${ }^{19}$, Z. Cui ${ }^{21,20}$, K. Dadzie ${ }^{9}$, C. Delgado ${ }^{28}$, S. Della Torre ${ }^{30}$, M.B.Demirköz ${ }^{2}$, L. Derome ${ }^{16}$, S. Di Falco ${ }^{37}$, V. Di Felice ${ }^{49,43}$, C. Díaz ${ }^{28}$, F. Dimiccoli ${ }^{38}$, P. von Doetinchem ${ }^{19}$, F. Dong ${ }^{33}$, F. Donnini ${ }^{49,43}$, M. Duranti ${ }^{35}$, A. Egorov ${ }^{9}$, A. Eline ${ }^{9}$, J. Feng ${ }^{9}$, E. Fiandrini ${ }^{35,36}$, P. Fisher ${ }^{9}$, V. Formato ${ }^{49,43}$, C. Freeman ${ }^{19}$, C. Gámez ${ }^{28}$, R.J. García-López ${ }^{25}$, C. Gargiulo ${ }^{14}$, H. Gast ${ }^{1}$, M. Gervasi ${ }^{30,31}$, F. Giovacchini ${ }^{28}$, D. M. Gómez-Coral ${ }^{19}$, J. Gong ${ }^{33}$, C. Goy ${ }^{3}$, V. Grabski ${ }^{29}$, D. Grandi ${ }^{30,31}$, M. Graziani ${ }^{35,36}$, S. Haino ${ }^{46}$, K.C. Han ${ }^{27}$, R.K. Hashmani ${ }^{2}$, Z.H. $\mathrm{He}^{18}$, B. Heber ${ }^{24}$, T.H. Hsieh ${ }^{9}$, J.Y.Hu ${ }^{6,7}$, M. Incagli ${ }^{37}$, W.Y. Jang ${ }^{12}$, Yi Jia ${ }^{9}$, H. Jinchi2 ${ }^{27}$, B. Khiali ${ }^{49,43}$, G.N. Kim ${ }^{12}$, Th. Kirn ${ }^{1}$, M. Konyushikhin ${ }^{9}$, O. Kounina ${ }^{9}$, A. Kounine9, V. Koutsenko9 , D. Krasnopevtsev9, A. Kuhlman ${ }^{19}$, A. Kulemzin ${ }^{9}$, G. La Vacca ${ }^{30,31}$,

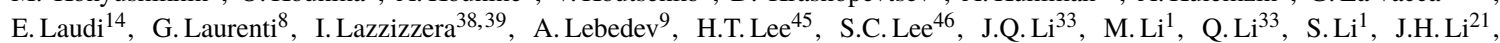

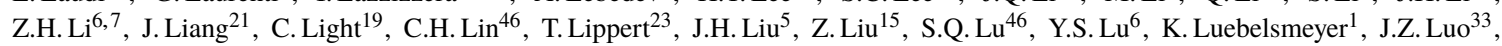

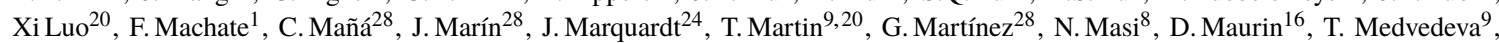
A. Menchaca-Rocha ${ }^{29}$, Q. Meng ${ }^{33}$, V.V.Mikhailov ${ }^{32}$, M. Molero ${ }^{28}$, P. Mott ${ }^{9,20}$, L. Mussolin ${ }^{35,36}$, J.Negrete ${ }^{19}$, N. Nikonov ${ }^{1}$, F. Nozzoli $^{38}$, A. Oliva ${ }^{8}$, M. Orcinha ${ }^{26}$, M. Palermo ${ }^{19}$, F. Palmonari ${ }^{8}$, M.Paniccia ${ }^{15}$, A. Pashnin ${ }^{9}$, M. Pauluzzi ${ }^{35,36}$, S. Pensotti ${ }^{30,31}$, H.D. Phan ${ }^{9}$, V. Plyaskin ${ }^{9}$, M.Poh1 ${ }^{15}$, S. Poluianov ${ }^{34}$, X. Qin ${ }^{9}$, Z.Y. Qu ${ }^{46}$, L. Quadrani ${ }^{8}$, P.G. Rancoita ${ }^{30}$, D. Rapin ${ }^{15}$, A. Reina Conde $^{25}$, E. Robyn ${ }^{15}$, S. Rosier-Lees ${ }^{3}$, A. Rozhkov ${ }^{9}$, D. Rozza ${ }^{30,31}$, R. Sagdeev ${ }^{10}$, S. Schael ${ }^{1}$, A. Schulz von Dratzig ${ }^{1}$, G. Schwering ${ }^{1}$, E.S.Seo ${ }^{11}$, Z. Shakfa ${ }^{2}$, B.S. Shan ${ }^{4}$, T. Siedenburg ${ }^{1}$, C. Solano ${ }^{9}$, J.W. Song ${ }^{21}$, X.J. Song ${ }^{22}$, R. Sonnabend ${ }^{1}$, L. Strigari ${ }^{41}$, T. Su ${ }^{22}$,

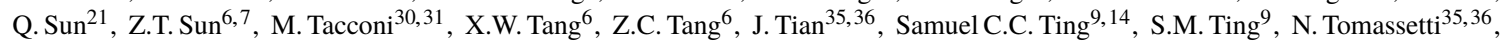
J. Torsti ${ }^{48}$, C. Tüysüz ${ }^{2}$, T. Urban ${ }^{9,20}$, I. Usoskin ${ }^{34}$, V. Vagelli ${ }^{40,35}$, R. Vainio ${ }^{48}$, M. Valencia-Otero ${ }^{47}$, E. Valente ${ }^{41,42}$, E. Valtonen ${ }^{48}$, M. Vázquez Acosta ${ }^{25}$, M. Vecchi ${ }^{17}$, M. Velasco ${ }^{28}$, J.P. Vialle ${ }^{3}$, C.X. Wang ${ }^{21}$, L. Wang ${ }^{5}$, L.Q. Wang ${ }^{21}$, N.H. Wang ${ }^{21}$, Q.L. Wang ${ }^{5}$, S. Wang ${ }^{19}$, X. Wang ${ }^{9}$, Yu Wang ${ }^{21}$, Z.M. Wang ${ }^{22}$, J. Wei ${ }^{15}$, Z.L.Weng ${ }^{9}$, H. Wu ${ }^{33}$, R.Q.Xiong ${ }^{33}$, W. Xu ${ }^{21,22}$, Q. Yan ${ }^{9}$, Y. Yang ${ }^{44}$,

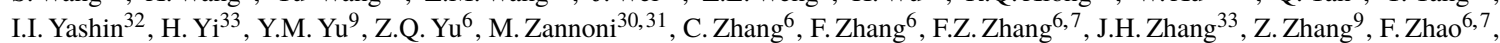

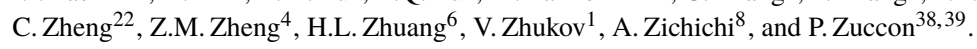

${ }^{1}$ I. Physics Institute and JARA-FAME, RWTH Aachen University, 52056 Aachen, Germany. ${ }^{2}$ Department of Physics, Middle East Technical University (METU), 06800 Ankara, Turkey. ${ }^{3}$ Univ. Grenoble Alpes, Univ. Savoie Mont Blanc, CNRS, LAPP-IN2P3, 74000 Annecy, France. ${ }^{4}$ Beihang University (BUAA), Beijing, 100191, China. ${ }^{5}$ Institute of Electrical Engineering (IEE), Chinese Academy of Sciences, Beijing, 100190, China. ${ }^{6}$ Institute of High Energy Physics (IHEP), Chinese Academy of Sciences, Beijing, 100049, China. ${ }^{7}$ University of Chinese Academy of Sciences (UCAS), Beijing, 100049, China. ${ }^{8}$ INFN Sezione di Bologna, 40126 Bologna, Italy. ${ }^{8}$ Università di Bologna, 40126 Bologna, Italy. ${ }^{9}$ Massachusetts Institute of Technology (MIT), Cambridge, Massachusetts 02139, USA. ${ }^{10}$ East-West Center for Space Science, University of Maryland, College Park, Maryland 207, USA. ${ }^{11}$ IPST, University of Maryland, College Park, Maryland 20742, USA. ${ }^{12}$ CHEP, Kyungpook National University, 41566 Daegu, Korea. ${ }^{13}$ CNR-IROE, 50125 Firenze, Italy. ${ }^{14}$ European Organization for Nuclear Research (CERN), 1211 Geneva 23, Switzerland. ${ }^{15}$ DPNC, Université de Genève, 1211 Genève 4, Switzerland, ${ }^{16}$ Univ. Grenoble Alpes, CNRS, Grenoble INP, LPSC-IN2P3, 38000 Grenoble, France. ${ }^{17}$ Kapteyn Astronomical Institute, University of Groningen, P.O. Box 800, 9700 AV Groningen, Netherlands. ${ }^{18}$ Sun Yat-Sen University (SYSU), Guangzhou, 510275, China. ${ }^{19}$ Physics and Astronomy Department, University of Hawaii, Honolulu, Hawaii 96822, USA. ${ }^{20}$ National Aeronautics and Space Administration Johnson Space Center (JSC), Houston, Texas 77058, USA. ${ }^{21}$ Shandong University (SDU), Jinan, Shandong, 250100, China. ${ }^{22}$ Shandong Institute of Advanced Technology (SDIAT), Jinan, Shandong, 250100, China. ${ }^{23}$ Jülich Supercomputing Centre and JARA-FAME, Research Centre Jülich, 52425 Jülich, Germany. ${ }^{24}$ Institut für Experimentelle und Angewandte Physik, Christian-Alberts-Universität zu Kiel, 24118 Kiel, Germany. ${ }^{25}$ Instituto de Astrofísica de Canarias (IAC), 38205 La Laguna, and Departamento de Astrofísica, Universidad de La Laguna, 38206 La Laguna, Tenerife, Spain. ${ }^{26}$ Laboratório de Instrumentação e Física Experimental de Partículas (LIP), 1649-003 Lisboa, Portugal. ${ }^{27}$ National Chung-Shan Institute of Science and Technology (NCSIST), Longtan, Tao Yuan, 32546, Taiwan. ${ }^{28}$ Centro de Investigaciones Energéticas, Medioambientales y Tecnológicas (CIEMAT), 28040 Madrid, Spain. ${ }^{29}$ Instituto de Física, Universidad Nacional Autónoma de México (UNAM), Ciudad de México, 01000 Mexico. ${ }^{30}$ INFN Sezione di Milano-Bicocca, 20126 Milano, Italy. ${ }^{31}$ Università di Milano-Bicocca, 20126 Milano, Italy. ${ }^{32}$ NRNU MEPhI (Moscow Engineering Physics Institute), Moscow, 115409 Russia. ${ }^{33}$ Southeast University (SEU), Nanjing, 210096, China. ${ }^{34}$ Sodankylä Geophysical Observatory and Space Physics and Astronomy Research Unit, University of Oulu, 90014 Oulu, Finland. ${ }^{35}$ INFN Sezione di Perugia, 06100 Perugia, Italy. ${ }^{36}$ Università di Perugia, 06100 Perugia, Italy. ${ }^{37}$ INFN Sezione di Pisa, 56100 Pisa, Italy. ${ }^{38}$ INFN TIFPA, 38123 Povo, Trento, Italy. ${ }^{39}$ Università di Trento, 38123 Povo, Trento, Italy. ${ }^{40}$ Agenzia Spaziale Italiana (ASI), 001 Roma, Italy. ${ }^{41}$ INFN Sezione di Roma 1, 00185 Roma, Italy. ${ }^{42}$ Università di Roma La Sapienza, 00185 Roma, Italy. ${ }^{43}$ INFN Sezione di Roma Tor Vergata, 00133 Roma, Italy. ${ }^{44}$ National Cheng Kung University, Tainan, 70101, Taiwan. ${ }^{45}$ Academia Sinica Grid Center (ASGC), Nankang, Taipei, 11529, Taiwan. ${ }^{46}$ Institute of Physics, Academia Sinica, Nankang, Taipei, 11529, Taiwan. ${ }^{47}$ Physics Department and Center for High Energy and High Field Physics, National Central University (NCU), Tao Yuan, 32054, Taiwan. ${ }^{48}$ Space Research Laboratory, Department of Physics and Astronomy, University of Turku, 20014 Turku, Finland. ${ }^{49}$ ASI Space Science Data Center (SSDC), 00133 Roma, Italy. 